\title{
Standardized phantoms for quantitative cardiac MRI
}

\author{
Katy Keenan", Karl F Stupic, Michael A Boss, Stephen E Russek \\ From 18th Annual SCMR Scientific Sessions \\ Nice, France. 4-7 February 2015
}

\section{Background}

Quantitative MR relaxometry techniques are increasingly used in cardiac MR applications, e.g. MOLLI for high resolution T1 mapping. To use these techniques in the clinic, we must understand how to make comparable measurements across vendor systems and software versions and compare results across sites. A standardized phantom used for regular quality control is one approach. The Biomagnetic Imaging program at the National Institute for Standards and Technology (NIST) has created phantoms for measuring $\mathrm{T} 1, \mathrm{~T} 2$, proton density in collaboration with ISMRM (Fig. 1) and diffusion in collaboration with RSNA-QIBA. In addition, a breast phantom including $\mathrm{T} 1$ and diffusion components is in development with UCSF. We propose a cardiac phantom focused on quantitative measurements of the myocardium pre- and post-contrast and the blood pool.

To test quantitative cardiac MR sequences, the phantom must mimic both the T1 and T2 relaxation properties in the same sample. The phantom must be stable, preferably for five years, and be reliably produced. Agarose gel is difficult to produce free of air bubbles and has a limited shelf life.

\section{Methods}

We think the relaxation times can be mimicked using paramagnetic salts and metallic oxides in aqueous solution. By our calculations for $1.5 \mathrm{~T}$, the post-contrast myocardium relaxation properties $(\mathrm{T} 1 \sim 200 \mathrm{~ms}, \mathrm{~T} 2 \sim$ $50 \mathrm{~ms}$ ) can be mimicked using an aqueous solution of $8.32 \mathrm{mM} \mathrm{NiCl} 2$ and $0.36 \mathrm{mM} \mathrm{MnCl} 2$ or by $0.25 \mathrm{mM}$ Feraheme solution. The pre-contrast myocardium and blood pool relaxation properties are more difficult. Based on experiments in our lab at $1.5 \mathrm{~T}$, the pre-contrast myocardium relaxation properties $(\mathrm{T} 1 \sim 1000 \mathrm{~ms}$,

\footnotetext{
NIST, Boulder, CO, USA
} any medium, provided the original work is properly cited. The Creative Commons Public Domain Dedication waiver (http:// creativecommons.org/publicdomain/zero/1.0/) applies to the data made available in this article, unless otherwise stated. 


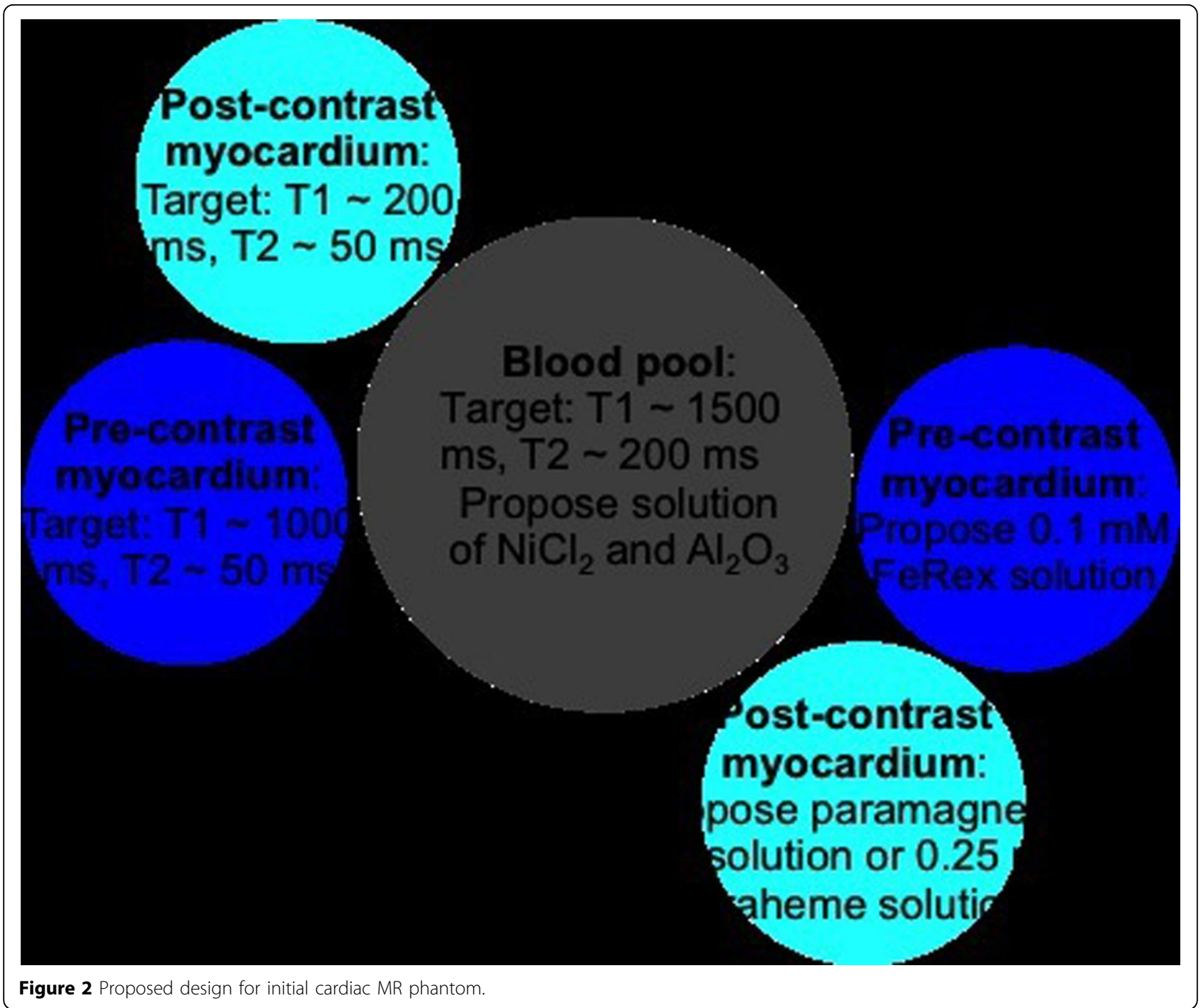

$23 \mathrm{~mm}$ ) for the blood pool and four smaller tubes (inner diameter $15 \mathrm{~mm}$ ) for the pre- and post-contrast myocardium (Fig. 2). Future design could be an injection molded object with three compartments to better match the geometry of the left ventricle and healthy to diseased myocardium wall thickness (6 to $15 \mathrm{~mm}$ ). In addition, a chest cavity material could surround future designs for proper loading of the coils.

\section{Conclusions}

Future design could be an injection molded object with three compartments to better match the geometry of the left ventricle and healthy to diseased myocardium wall thickness (6 to $15 \mathrm{~mm}$ ). In addition, a chest cavity material could surround future designs for proper loading of the coils.

\section{Funding}

N/A.

Published: 3 February 2015

doi:10.1186/1532-429X-17-S1-W36

Cite this article as: Keenan et al:: Standardized phantoms for

quantitative cardiac MRI. Journal of Cardiovascular Magnetic Resonance 2015 17(Suppl 1):W36. 